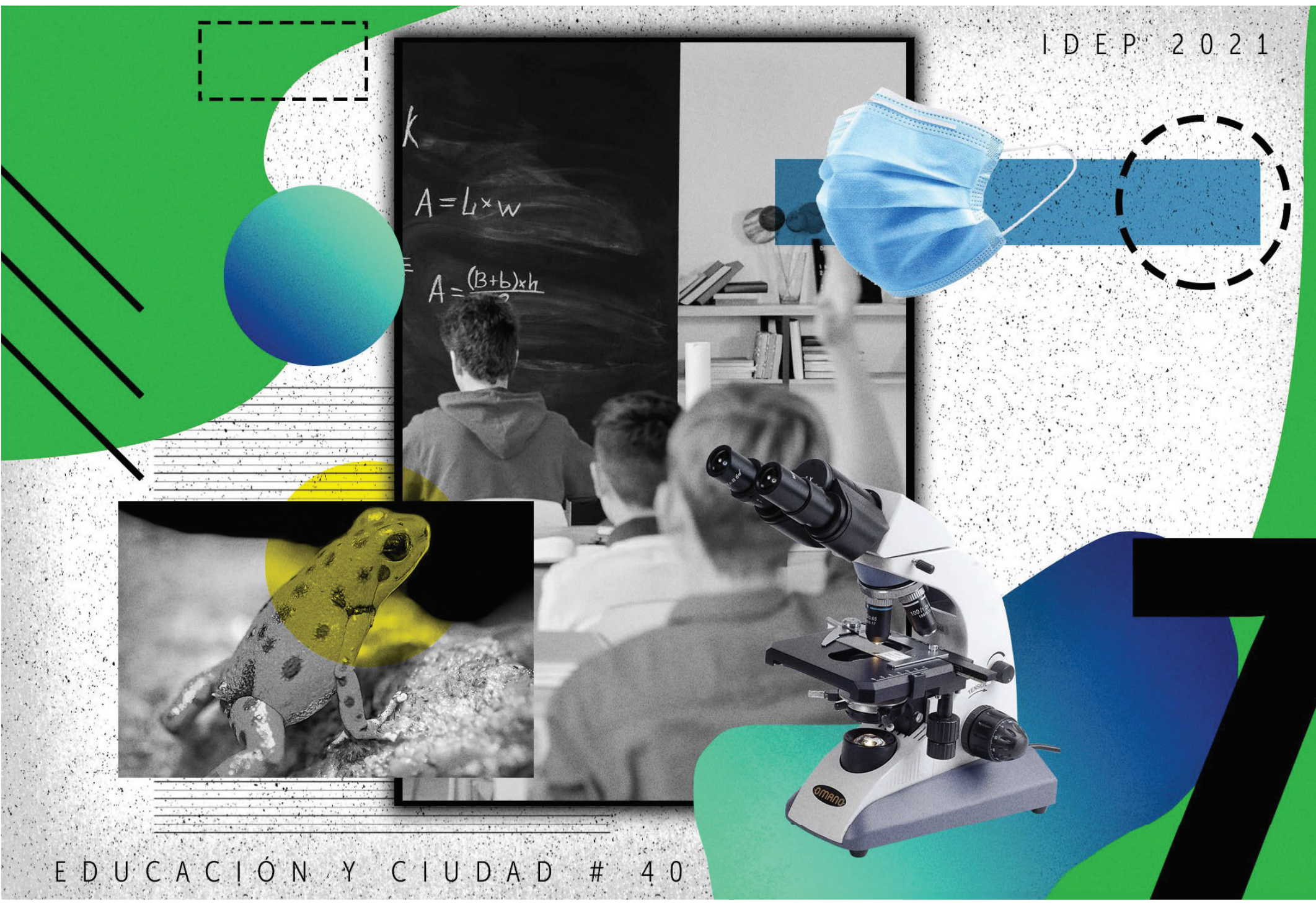

\title{
Prácticas y reflexiones sobre el ambiente: miradas desde la formación de maestros de biología ${ }^{1}$
}

PRACTICES AND REFLECTIONS ON THE ENVIRONMENT: VIEWS FROM THE TRAINING OF BIOLOGY TEACHERS

PRÁTICAS E REFLEXÕES SOBRE O AMBIENTE: VISÕES DA FORMAÇÃO DE PROFESSORES DE BIOLOGÍA 


\section{Deysi Serrato Rodríguez ${ }^{2}$}

2. Licenciada en Biología; Especialista en Pedagogía; Magíster en Educación; Doctoranda en Educación, Doctorado Interinstitucional en Educación, Énfasis en Historia de la Educación, Pedagogía y Educación Comparada; Profesora ocasional, Departamento de Biología, Universidad Pedagógica Nacional; Cvlac: http://scienti.colciencias.gov.co:8081/cvlac/visualizador/generarCurriculoCv. do?cod_rh=0001543875; ORCID: https://orcid.org/0000-0002-9071-4589; correo electrónico: dlserrator@pedagogica.edu.co

Citar artículo como:

Serrato, D. (2021, Enero-Junio). Prácticas y reflexiones sobre el ambiente: miradas desde la formación de maestros de biología. Revista Educación y Ciudad, No. 40, pp.113-127. //doi.org/10.36737/01230425. $\mathrm{n} 40.2021 .2460$

DOI: https://doi.org/10.36737/01230425.n40.2021.2460

Fecha de recepción: 4 de agosto de 2020 / Fecha de aprobación: 4 de noviembre de 2020

\section{Resumen}

El artículo presenta algunas reflexiones y prácticas vinculadas a los futuros maestros de biología de la Universidad Pedagógica Nacional, particularmente en relación con el ambiente y sus propuestas para abordarlo en la escuela. Entre otras, se posicionan comprensiones alrededor del ambiente en tanto recurso, biosfera, experiencia y capital natural, tejiendo relaciones con la ecología, la economía y la política; igualmente, se sustenta la importancia de las ciudadanías ambientales para el cuidado y protección del ambiente y la naturaleza para, finalmente, proponer una mirada distinta desde la enseñanza ambiental y la experiencia de los sujetos.

Palabras clave: Ambiente, maestro, enseñanza ambiental, pedagogía, experiencia.

\section{Abstract}

The article presents some reflections and practices of the future biology teachers of the National Pedagogical University in relation to the environment and their teaching proposals at school. Understandings are positioned around the environment as a resource, biosphere, experience, natural capital, among others, weaving relationships with ecology, economics and politics. Similarly, environmental citizenships are important for the care and protection of the environment and nature. Finally, a different view is proposed from the environmental teaching and the experience of the subjects.

Keywords: Environment, teacher, environmental education, pedagogy, experience.

\section{Resumo}

$\mathrm{O}$ artigo apresenta algumas reflexões e práticas dos futuros professores de biologia da Universidade Nacional Pedagógica em relação ao meio ambiente e suas propostas para uma abordagem escolar. Os entendimentos estão posicionados em torno do meio ambiente como recurso, biosfera, experiência, capital natural, entre outros, tecendo relações com ecologia, economia e política. Da mesma forma, a importância das cidadanias ambientais para o cuidado e a proteção do meio ambiente e da natureza é sustentada. Finalmente, uma perspectiva diferente é proposta a partir do ensino ambiental e da experiência das pessoas.

Palavras-chave: Ambiente, professor, educação ambiental, pedagogia, experiencia

\footnotetext{
${ }^{1}$ Las reflexiones presentadas en este artículo hacen parte del trabajo de grado de la Maestría en Educación de la Universidad Pedagógica Nacional, titulado La investigación sobre lo vivo y la vida como práctica de gobierno en el proyecto curricular Licenciatura en Biología de la Universidad Pedagógica Nacional (2016).
} 
Problematizaciones alrededor del ambiente y su abordaje en la escuela: miradas desde la formación de maestros de biología

Sin duda alguna, la cuestión del ambiente se $\checkmark$ posiciona como elemento de fundamental importancia en las dinámicas contemporáneas de vida; tal es el motivo para abordarle, en este caso particular, tanto en la escuela, como en las lógicas de formación de maestros de biología. Desde dichos escenarios se construyen problematizaciones que interrogan prácticas activistas y ecologistas sin tenido mayor incidencia en las relaciones de los sujetos con el ambiente, aunque se manifiestan en sus comportamientos hacia el mismo y en la propia persona.

Del mismo modo, es importante cuestionar el abordaje del ambiente desde la perspectiva de revisar los proyectos que muchas veces no impactan el currículo escolar, lo cual se suma a la cuestión de designar dicha tarea a los maestros de ciencias, específicamente a los de biología, en el supuesto de que son ellos quienes tienen los conocimientos asociados al ambiente y por tanto puede propiciar su cuidado y protección. Estos lugares comunes dejan en evidencia lo que Morin (2007) llama el paradigma de la simplicidad, el cual consiste en pensar el mundo de forma fragmentada, aislada y separada, desconociendo las múltiples relaciones tejidas entre todos los niveles de complejidad propios de la realidad.

Ello destaca la importancia de comenzar a pensar y desplegar prácticas relacionadas con el ambiente desde la complejidad, pero, sobre todo, desde una mirada sistémica que permita comprender que éste no se reduce a eso "verde" o "natural" que debe ser cuidado o gestionado, sino que se constituye a partir de un entramado de relaciones entre factores biológicos, sociales, culturales, políticos y económicos, que condicionan nuestra mirada frente al mismo y, por tanto, su abordaje en la escuela y desde de la formación de maestros de biología; podría pensarse como una dimensión de la subjetividad, en tanto implica un reconocimiento de sí mismo, de los otros y de lo otro, lo cual, partiendo de un ejercicio de alteridad, y en clave de la pedagogía, podría brindar elementos para lograr el tan anhelado cambio frente a las prácticas de consumo, cuidado y explotación de lo que se ha denominado ambiente.

Partiendo de dichas problematizaciones, el presente escrito busca visibilizar algunas reflexiones, construcciones y propuestas a propósito del ambiente, elaboradas por algunos maestros en formación de la Licenciatura en Biología de la Universidad Pedagógica Nacional (UPN), brindando así múltiples perspectivas para repensar las prácticas asociadas a lo ambiental. Para el caso, se destaca la propuesta de la enseñanza ambiental como escenario de problematización y posibilidad para situar el ambiente desde el encuentro de las experiencias de los sujetos.

\section{Las cuestiones de método}

Para visibilizar lo propuesto, se recurre a la constitución de un archivo que, en tanto herramienta metodológica, remite a prácticas y al conjunto de reglas empleadas para establecer el debate en una sociedad determinada, cuáles son los enunciados válidos, qué individuos o grupos tienen acceso a determina- 
dos tipos de discurso y cómo están institucionalizadas las relaciones de poder entre quienes lo emiten o lo reciben. Así mismo, trabajar desde el archivo posibilita trasladarse del lugar del experto y asumir todos los documentos con la misma relevancia, destacando el saber que circula desde ellos. Dicho esto, la constitución del archivo se consolida mediante la revisión de documentos, entendiéndolos:

[...] no tanto como fuente, sino como registro de prácticas [...] es un registro donde ya se ha hablado desde un ejercicio de saber, cruzado por instituciones, discursos, sujetos y prácticas diferentes, desde ahí que se asuma el documento visto desde su discurso, como un tejido relacional (Zuluaga, 1999, p. 18).

En esa vía, el archivo constituido para estas reflexiones y discusiones se fundamenta en los trabajos de grado y proyectos de práctica pedagógica de los maestros en formación de la Licenciatura en Biología de la Universidad Pedagógica Nacional; dichos documentos pasan por el ejercicio de la "tematización” a través de fichas temáticas y matrices, cuyo objetivo principal es:

[...] registrar la información temática de los documentos claves identificados durante la investigación. Se trata de un procedimiento metodológico que busca desarticular los textos en sus temáticas centrales o principales para posibilitar, de esa manera, cruces horizontales entre documentos de diverso tipo y nivel de los cuales deberán salir agrupamientos temáticos en los que sea posible ubicar cada uno de los documentos trabajados (Castro, 2007, p. 2).

Este ejercicio logra dar cuenta de la dispersión documental, permitiendo observar los enunciados diseminados en los diferentes registros. Cuando se rompe la unidad de los documentos rastreados, visibilizando tal dispersión de los enunciados de una época, se hace posible dejar atrás la idea de continuidad y causalidad, reconociendo los movimientos del saber. Junto a ello, la lectura hipertextual permite desarticular los textos para luego establecer otras relaciones entre ellos; finalmente, será posible hablar de una identificación de regularidades y discontinuidades, donde las fichas temáticas y matrices se constituyen como elementos indispensables, pues a través de su lectura y de las relaciones que se logran establecer, se llegará a identificar las regularidades discursivas y visibilizar los enunciados que orientan la estructura de un texto, estableciendo otro tipo de tejido y lectura.

\section{Multiplicidad de miradas frente al ambiente, desde la formación de maestros de biología de la UPN}

En el marco de la formación de maestros de biología en la Universidad Pedagógica Nacional, específicamente para los trabajos de grado realizados entre 2000 y 2015 , se hace evidente la idea del ambiente como un escenario que deja entrever las relaciones entre lo vivo y la vida. En dichas relaciones se sitúa la cuestión ambiental como idea recurrente, al punto de encontrar una serie de comprensiones desde lo planteado por Sauvé (1994), quien establece que las representaciones alrededor del tema pueden ser diversas y que de ellas depende el comportamiento asumido por los sujetos. Así, se destaca el ambiente como problema por solucionar, recurso para administrar, naturaleza para apreciar y respetar, biosfera para vivir juntos, medio de vida para conocer, escenario comunitario para participar y "holos"; con tales ideas, se sitúa regularmente el acercamiento al 
ambiente en tanto recurso, problema a solucionar, biosfera y naturaleza para apreciar, hallando que:

En cuanto al ambiente como recurso, se menciona: Los valores y las tradiciones culturales como recurso para vender a los turistas. Elementos biofísicos como el Río Amazonas, el paisaje, la luna, los animales, entre otros. Que son ofrecidos como atractivo para ser contemplados en los senderos y otros recorridos, por los turistas (Rodríguez, 2011, p. 87).

En términos delambiente como naturaleza y biosfera, se hallan expresiones que remiten a pensarle como un conjunto de interrelaciones entre lo biótico y lo abiótico desde una constante convivencia armónica; de igual forma, se sitúa la noción de ambiente desde las relaciones que establecen lo sujetos con, entre otros aspectos, la escuela, la comunidad, el suelo, el entorno o la tierra:

Es evidente la manera como los estudiantes, frente al concepto ambiente, emiten una serie de razonamientos que se vinculan directamente con las vivencias del contexto social donde se desarrollan, reflejando la relación que establecen con la escuela, su familia, los amigos, además de algunos valores asociados a la conservación biológica de ciertos elementos de la naturaleza, en cuanto a las categorías de análisis las relaciones sociales establecen vínculos entre las vivencias y la realidad de un ambiente social donde se involucran factores de tipo violento, marcados quizá por la situación que atraviesa el país y, sin ir más lejos, por la inseguridad del sector en donde se vive (Bilbao y Garzón, 2003, pp. 66-67).

El ambiente se instala entonces como un sistema constituido por factores naturales, culturales y sociales interrelacionados entre sí, que condicionan la vida del hombre, a la vez que son constantemente modificados e intervenidos por éste. En tal sentido, como señalan Bejarano y Mora (2008), el ambiente tiene un doble valor: es fuente de recursos que alimenta la humanidad y es el ámbito donde ella evoluciona y desarrolla sus formas culturales, que elabora como respuesta a la naturaleza y a su propia condición de ser vivo.

Por otro lado, de la mano de Eschenhagen (2010) se proponen tres formas de entender el ambiente, donde la crítica a la racionalidad instrumental y económica se hace relevante; el primer acercamiento es el ambiente como objeto, cuya base epistemológica parte del positivismo que fragmenta, cosifica y objetiviza los elementos del entorno, incluyendo al ser humano; la segunda perspectiva es el ambiente como sistema, su fundamento es la Teoría General de Sistemas de Ludwig Von Bertalanffy, donde se afirma que las interacciones entre objetos son interdependientes a través de las relaciones entre ellos $\mathrm{y}$, finalmente, la tercera comprensión del ambiente se da desde la crítica a la visión dominante, relacionada con unas formas de apropiación del medio en clave de la necesidad de cuestionar los presupuestos modernos.

De este modo, resulta difícil y poco conveniente pensar en una sola comprensión del ambiente, debido a la multiplicidad de miradas y relaciones establecidas a su alrededor; sin embargo, más allá de las definiciones, o de su delimitación, se trata de reconocer e interrogar cuáles son las prácticas construidas por los sujetos en torno al mismo, más aún cuando se despliegan diversidad de estrategias y acciones para generar cambios en los modos de relación con lo ambiental que, sin embargo, parecen no tienen la incidencia esperada. 


\section{La educación ambiental: ¿ambiente como problema a solucionar?}

Vista como problema para solucionar, la noción de ambiente remite a una explotación indiscriminada de recursos que pone en riesgo las dinámicas de la biodiversidad, pues en ella se establece que las acciones del hombre afectan de forma contundente, no solo su entorno, sino el de miles de organismos que lo comparten. Una de las posibles causas de este fenómeno de destrucción masiva para el mundo de lo vivo, puede entenderse desde el pensamiento "especista" que envuelve en la actualidad a la humanidad; bajo el argumento de ciertas capacidades como la racionalidad, el lenguaje y un supuesto origen divino, esta postura sitúa a la especie humana en un grado superior al de las demás formas de vida, contemplándolas como meros recursos a su disposición. Así, teniendo en cuenta lo anterior, la educación es establecida como estrategia para enfrentar las problemáticas ambientales relacionadas con la administración de recursos, pues:

[...] no solo responde a demandas de orden pedagógico, sino que se interconecta con aquellos problemas pertinentes, para este caso, la crisis y deterioro ambiental, en conexión con esferas sociales, políticas, económicas y culturales, donde el discurso del desarrollo ha tomado vigor y fuerza para ofrecer otro tipo de estrategias de control, tales como la pobreza y el hambre, en tanto establecen nuevos mecanismos de intervención de la vida (Cárdenas, 2015, p. 134).

En ese sentido, desde las problematizaciones propuestas por Lastra y Ramírez (2012), se encuentra la educación ambiental como solución para los problemas en los lazos tejidos por el hombre con su am- biente, y como proceso que le permite comprender las relaciones de interdependencia con su entorno, teniendo en cuenta las dimensiones biofísica, social, política, económica y cultural, en donde la comunidad en general genera actitudes de valoración y respeto por el ambiente, con el fin de aportar al mejoramiento de las condiciones de vida de la misma.

Por consiguiente, la Educación Ambiental debe ser un proceso de enseñanza y aprendizaje, integrador, continuo, dinámico y permanente, mediante el cual los individuos se socializan y resocializan en una serie de experiencias, percepciones, conocimientos, habilidades, actitudes, valores y capacidades relacionadas con el cuidado y la conservación de los recursos naturales (Poveda, 2009).

Al respecto, desde sus prácticas, los maestros en formación proponen una serie de enfoques frente a la Educación Ambiental que permiten visibilizar cómo se despliega lo vivo, a propósito de una preocupación por su gestión en tanto recurso, pero también por las acciones a fomentar desde el campo educativo para estar en armonía con la naturaleza. En esa medida, López y Pérez (2010) llevan a destacar tres enfoques: el naturalista, que hace énfasis en la ecología y el estudio de las relaciones entre un organismo y su entorno; el integrador, que busca dar una información holística interdisciplinaria sobre las relaciones cultura-naturaleza y sobre los problemas surgidos de ella; y el de enseñanza aplicada, que apuesta por analizar un problema, sus causas, consecuencias y soluciones.

Desde esta óptica, los enfoques expuestos permiten enunciar la forma en que lo vivo, entendido como los organismos y sus relaciones con el entorno, es posicionado como objeto de cuidado, siendo 
la educación ambiental una estrategia cuyo fin es aportar a la vida desde su protección y conservación. Así, la educación ambiental se concibe como una apuesta interdisciplinar, en tanto teje relaciones de diversos saberes, principalmente desde la conservación y la ecología:

Por consiguiente, para comprender lo ambiental, un primer paso lo constituye el conocimiento de la ecología, la manera como en general interactúan los seres vivos con su entorno. Un segundo paso para comprender lo ambiental es profundizar el estudio de la sociedad y la cultura. En pocas palabras, el concepto ambiente abarca el estudio de la estructura y funcionamiento de los ecosistemas, la forma en la que se establecen las relaciones sociales y la manera cómo interactúan estos dos sistemas (Piñeros, 2008, p. 29).

Es tal la fuerza de la ecología en la lectura de lo ambiental que, en el marco de la escuela, se asume su enseñanza como dirigida a que los estudiantes expliquen las ventajas de la conservación del medio ambiente, sepan identificar problemas vinculados con la contaminación y destrucción de su entorno, hallen posibles soluciones y manifiesten y se identifiquen con valores que propendan por el respeto a la vida y el ambiente. Sin embargo, es importante problematizar la apropiación de la ecología en la escuela desde lo ambiental, pues se sospecha que, a pesar de retomar elementos de ella, se cae en su reducción, al pensarles desde el activismo implícito en actividades como el reciclaje y la reutilización, las cuales pueden funcionar como acciones desde la inmediatez, pero posiblemente no aportan significativamente a pensar de otras maneras las relaciones con el ambiente.

Ahora bien, es pertinente reconocer que, desde los discursos de educación ambiental y las construccio- nes más recientes sobre el ambiente, se han venido desplegando propuestas de interés como la pedagogía ambiental (Torres, 2015), la otredad ambiental y la complejidad territorial (Paz, 2009), o la dimensión filosófica de la ética (González, 2017), entre otros que, sin duda, pueden mover de los lugares comunes el abordaje del ambiente en la escuela, problematizados hasta este punto, potenciando la construcción de otras relaciones entre los sujetos y lo ambiental, mucho más significativas y "experienciales”. Sin embargo, vale la pena interrogar: ¿Qué condiciones son necesarias para posicionar estas propuestas en la escuela?; ¿desde la formación de maestros de biología, cómo se podrían construir los escenarios para materializar tales planteamientos?

\section{Hacia la formación de ciudadanía ambiental}

Desde la preocupación por el ambiente y su cuidado, las formas de comprensión sobre lo vivo sientan las condiciones para pensar en la constitución de sujetos que viabilicen las acciones promulgadas por la educación ambiental; desde allí surge la urgencia de formar ciudadanos ambientales capaces de reconocer situaciones problemáticas que afectan de una manera u otra su medio; esperando que dicha formación desde la escuela lleve a los estudiantes a establecer estrategias para buscar soluciones y mejorar su calidad de vida y del medio que les rodea; entonces:

[...] educar para la ciudadanía ambiental implica combatir contra una serie de elementos contradictorios que existen en el orden en que nos desempeñamos como sujetos sociales, que nos influye sobre cómo actuar en relación con el ambiente, y esos elementos se relacionan con la forma en como vemos el ambiente, cómo nos relacionamos con 
él y, sobre todo, tiene que ver con nuestros estilos de vida, es decir que se lucha por "lograr que las personas de los distintos países asuman de manera clara y concreta un papel equilibrado en su actuación frente a la naturaleza, así como frente a los demás seres humanos" (Gómez y Acuña, 2009, p. 52).

De este modo, la educación para la ciudadanía ambiental debe estar enmarcada dentro de una política ambiental y cultural que favorezca la construcción de relaciones apropiadas entre el hombre y el medio, lo cual implica el despliegue de un proceso de suma complejidad (González, 2003). Ahora bien, en el marco de los discursosidentificados por los futuros maestros de biología, la ciudadanía ambiental se relaciona con elementos tales como la identidad, en tanto construcción colectiva, singular y local, el ejercicio de la democracia, la participación en la toma de decisiones frente a lo ambiental y el empoderamiento de las dinámicas que atraviesan los distintos territorios.

Sin embargo, la idea de ciudadanía ambiental permite pensar en procedimientos de gobierno sobre la vida de los sujetos, condicionados por unas formas de comprensión de lo vivo a propósito del ambiente, las cuales se encuentran cruzadas por las nociones de cuidado y protección, hecho que resulta estratégico, pues la idea es gestionar y regular la explotación o utilización del ambiente como recurso; de ahí la posibilidad de sospechar que aunque la preocupación por el ambiente pasa por una apreciación y valoración de lo vivo en términos de su cuidado, también implica apostar por las lógicas del desarrollo que funcionan en el marco del neoliberalismo. Esta relación permite evidenciar algunos juegos de verdad a propósito del ambiente y el desarrollo.
Con dichos presupuestos, es importante ampliar la mirada, pues la noción de ciudadanía ambiental atraviesa debates vinculados a sus connotaciones jurídicas, sus vínculos con la perspectiva de gestión ambiental y, sobre todo, por la relación de exterioridad que plantea frente al ambiente como un todo. En esa vía, se encuentran propuestas pensadas desde otros lugares, para el caso, es posible destacar la noción de meta-ciudadanías ecológicas (Gudynas, 2009), que propone perspectivas plurales y colectivas para cuestionar las miradas modernas del ambiente, y posicionar ontologías relacionales donde se involucren distintas cosmovisiones, dando lugar a construcciones biorregionales más particulares y menos generales.

\section{Ambiente y capital natural: ¿Gestión de lo vivo?}

Gran parte de las prácticas desplegadas frente al ambiente por los maestros en formación de la Licenciatura en Biología, cuestionan la apropiación del mismo en tanto recurso o capital, pues se encuentran frecuentemente con una urgencia por la gestión de lo natural o lo ambiental desde las dinámicas del desarrollo; tanto así, que se logran evidenciar planteamientos asociados al ambiente como capital natural. De acuerdo con Costanza y Daly (1992), el capital natural está conformado por los recursos naturales, entendidos como las riquezas de la naturaleza que generan rendimientos económicos, por ello, los recursos están encaminados a funciones como la inversión, consumo y producción; tal comprensión termina validando nuevas formas de expropiación y apropiación de lo natural, pues: 


\begin{abstract}
Actualmente el mundo atraviesa por un proceso de creciente globalización [...] En este contexto las empresas multinacionales se han dado cuenta del valor que poseen las semillas nativas por la diversidad genética de estas $\mathrm{y}$, por eso, han puesto sus ojos en ellas como un nuevo objeto de comercio, pero para asegurarlo necesitan construir mecanismos para que los agricultores solo realicen la siembra de las semillas tratadas previamente por las empresas, demeritando el valor de las semillas como legados culturales de los pueblos y creando leyes que prohíban la siembra de semillas nativas (Aguirre, 2015, p. 14).
\end{abstract}

Así, se establece la lógica de patentes en donde los bienes comunes, en este caso, lo natural, son expropiados por un aparato jurídico para convertirlos en mercancías. Se trata, pues, del proceso a través del cual un bien común se convierte en un bien privado que, como tal, queda inscrito en un sistema de regulaciones y normas que otorgan derechos exclusivos a los dueños de las licencias, y sanciones para quienes las violan. Pero ¿qué entender como bien común? Desde la perspectiva de Michelini (2007), se trata de aquellos bienes materiales e inmateriales no circunscritos a una persona, sino a todos y cada uno de los miembros de la sociedad; entre esos bienes suelen contarse elementos básicos para la supervivencia humana, como el aire y el agua, entre otros.

Desde esta perspectiva, la naturaleza y el ambiente, en tanto recursos disponibles y expresiones de lo vivo, se posicionan como objetos de regulación, posicionando la idea de una administración y gobierno de lo natural/ambiental en pro del desarrollo, aspecto que se liga con la propuesta de eco-gubernamentalidad planteada por Astrid Ulloa y retomada por Valencia y Tobar (2013), en términos de comprender el ambiente como objeto, no solo de conocimiento, sino de producción y gobierno:

\begin{abstract}
La ecogubernamentalidad ha sido definida entonces como "todas las políticas, los discursos, los conocimientos, las representaciones y las prácticas ambientales (locales, nacionales y transnacionales) que interactúan con el propósito de dirigir a los actores sociales (cuerpos verdes) a pensar y a comportarse de maneras particulares hacia fines ambientales específicos (desarrollo sostenible, seguridad ambiental, conservación de la biodiversidad, acceso a recursos genéticos, entre otros)" (Ulloa, 2004, XLII). En este contexto se ubican las actuales luchas por la apropiación de la biodiversidad en tanto campo de alta conflictualidad en el cual se debate el control de los espacios biofísicos y socio-culturales (Valencia y Tovar, 2013, p. 13).
\end{abstract}

Es desde esta perspectiva que se abre todo un campo de problematización alrededor de los discursos contemporáneos sobre el ambiente, y se sientan sospechas a propósito del funcionamiento de ciertos juegos de verdad en relación con su cuidado y protección, los cuales pueden condicionar la constitución de algunos estilos de vida en los sujetos y es necesario cuestionarles, pues algunos elementos llevan a pensar que lo ambiental, en sus lógicas de funcionamiento actuales, busca potenciar el desarrollo de las naciones desde una gestión y administración eficaz de los recursos y el ambiente en general.

\section{La propuesta desde la formación de maestros de biología: la enseñanza ambiental}

Desde la pedagogía, hablar de enseñanza implica situarla como incitadora del pensamiento, como el espacio relacional donde, a través del encuentro con otros, se logran construir experiencias que inciden en los modos de pensar y actuar de los sujetos. Así, inquietarse y construir posibilidades para asumir el ambiente de otro modo, significa deslocalizar 
y problematizar los discursos que se han ido legitimando desde lugares comunes alrededor del tema; en ese sentido, deben ser cuestionados asuntos como la problemática ambiental y el despliegue de estrategias desde lo educativo, no por las dinámicas de vida que tratan de intervenir y cambiar, sino por sus lecturas de ellas.

Al respecto, Leff (2004) plantea la problemática ambiental como resultado de una crisis de la racionalidad del mundo moderno en la que, desde una perspectiva científica y económica, valida la destrucción de la naturaleza en nombre del desarrollo y el bienestar; en consecuencia, la crisis ambiental corresponde a la crisis del efecto del conocimiento sobre el mundo. En este caso, se alude a la ciencia como forma de dominante del saber, que fragmenta y le desestructura con la pretensión de comprender, controlar y predecir los fenómenos, para mantener un estilo de vida considerado como deseado.

En sintonía con ello, Eschenhagen (2009) permite reconocer la causa última del problema en la visión dominante del mundo en la modernidad, que remite a tres crisis evidentes hoy día: la "crisis ambiental", representada por la degradación general del entorno; la "crisis social-civilizatoria", evidente en todo aquello que tiende a degradar a la humanidad (violencia, desempleo, condiciones de trabajo precarias, entre otros); y la "crisis intelectual", donde los marcos y métodos establecidos por la ciencia moderna son cuestionados, revisados o desechados cada vez con mayor insistencia, al reconocer que no ofrecen explicaciones y soluciones satisfactorias a un panorama contemporáneo cada vez más complejo (Lastra, 2013). Dichos planteamientos, permiten ver que las actuales prácticas asociadas al ambiente pueden constituirse como el resultado de:
[...] las formas de conocimiento a través de las cuales la humanidad ha construido el mundo y lo ha destruido por su pretensión de universalidad, de generalidad y de totalidad; por su objetivación y cosificación del mundo (Leff, 2007) pero, ante todo, es un asunto de sujetos, que tiene que ver con el sentir, con el pensamiento, las vivencias y las historias de vida de cada persona que vive y camina en el mundo (Lastra, 2013, p. 12).

Partiendo de tales presupuestos, se destaca la posibilidad de asumir el ambiente desde la experiencia construida por los sujetos alrededor del mismo, lo cual implica enunciar que la experiencia no es un acto de comprobación, pues se aparta de lo objetivo;por el contrario, se trata de un "acto de transformación de sí mismo, es decir, es estética, y como modificación de los otros no sólo es subjetiva, sino ética, y como transformación de la realidad es política” (Barragán, 2011, p. 10); así, entonces:

[...] el sujeto hace la experiencia de algo, pero, sobre todo, hace la experiencia de su propia transformación. De ahí que la experiencia me forma y me transforma. De ahí la relación constitutiva de formación [...] De ahí que el sujeto de la experiencia no sea el sujeto del saber, o el sujeto del poder, o el sujeto del querer, sino el sujeto de la formación y de la transformación (Larrosa, 2011, p. 17).

De este modo, tal como formularon Barajas y Villamil (2015), el ambiente se refleja a través de una experiencia de vida y, por tanto, son los sujetos quienes lo transforman a través de sus actividades y de su propio conocimiento. De esta forma, asumir el ambiente como experiencia permite:

[...] si se entiende el ambiente como una experiencia singular que se valida a través del pensamiento, no sería pertinente hablar de Educación Ambiental desde la perspectiva de pensamiento complejo [...] se plantea hablar de una enseñanza ambiental (Gómez y Bermúdez, 2015, p. 54). 
Enseñanza Ambiental que se erige como propuesta desde Vargas (2014), Lastra y Ramírez (2013) y Lastra (2013), como una oportunidad de pensar, asumir y relacionarse con el ambiente partiendo de la subjetividad y la experiencia. Así, desde un marco investigativo, es posible posicionar la enseñanza ambiental como fundamento para cuestionar los modos en que se ha abordado la educación ambiental en la escuela, dando paso al debate, las preguntas y la construcción colectiva y particular mientras, al tiempo, se ponen en duda los discursos generalistas que pretenden brindar soluciones a modo de receta frente a las dinámicas ambientales legitimadas. Para lograrlo, se aboga por una mirada interdisciplinar que supere las barreras impuestas por cada área y converger en el cuestionamiento y la construcción realizada por los sujetos frente al ambiente, logrando así una mirada desde la complejidad.

Posicionar la Enseñanza Ambiental aporta un nuevo sentido para abordar el eje ambiental en la escuela, desde la perspectiva de que el acto de enseñar en los diferentes contextos implica resignificar los contenidos de seminarios, programas de formación, pensamientos y experiencias asociados al tema, y abrirse a la posibilidad de abordarlos sin encerrarse en el activismo que ha caracterizado tradicionalmente a la Educación Ambiental. En ese sentido, como afirman Gómez y Bermúdez (2015), la Enseñanza Ambiental pretende reformar el pensamiento sobre el asunto ambiental, encontrando un llamado a superar la fragmentación de los saberes y la incapacidad de articularlos entre sí.

Así, la enseñanza ambiental puede constituirse en una de las tantas propuestas desplegadas por los maestros desde su práctica pedagógica y desde la problematización de las dinámicas escolares y los saberes que circulan en el campo educativo, para generar otros modos de relación entre sus estudiantes y lo que les rodea. De fondo, el asunto ambiental no se trata de una preocupación por las estrategias y métodos más efectivos para frenar el cambio o la crisis ambiental, ni de la forma de educar mejor al respecto, sino de lograr una transformación en el pensamiento frente a las dinámicas de vida actuales, reposando en otros modos de relación consigo mismo, con los otros y lo otro; desde allí, como saber de los maestros, la pedagogía tendría mucho que aportar.

\section{Conclusiones}

Las reflexiones y construcción frente al ambiente, elaboradas en el marco de la formación de maestros de biología de la Universidad Pedagógica Nacional, permiten una mirada poliédrica del mismo, centrada en reconocer los lugares comunes asociados al tema (crisis ambiental, problemática ambiental, ambiente como recurso, ambiente como capital para gestionar, etc.), los cuales se han instalado en la dinámica escolar; al tiempo, facilitan cuestionar e interrogar dichos aspectos para presentar otras miradas a propósito de lo ambiental, en este caso, desde la apuesta por la subjetividad y la construcción de experiencias cuyo origen es la pedagogía, elementos que se recogen y amplían al pensar en la Enseñanza Ambiental.

Así, es importante problematizar por qué en el escenario de la escuela no han tenido lugar las diversas construcciones actuales alrededor del ambiente, situadas, entre otras, desde posturas filosóficas, éticas, sociales y culturales, que resultan potentes para cuestionar el activismo y ecologismo evidentes en las aulas desde los paradigmas clásicos de educación 
ambiental, por qué se sigue replicando una serie de prácticas que han demostrado no ser funcionales.

Finalmente, el asunto de lo ambiental no se trata del despliegue de numerosas estrategias, métodos o proyectos para atender la denominada crisis ambiental o las problemáticas ambientales, aquello denominado "verde"; por el contrario, se podría pensar en la posibilidad de construir escenarios interdisciplinares de trabajo, que permitan develar la complejidad de lo ambiental, su singularidad, desde los distintos contextos y territorios, apostando por un cambio en los modos de relación entre los sujetos; se trata de una transformación en la racionalidad y el pensamiento, donde, para el caso de la enseñanza y la formación, la pedagogía, como saber del maestro, podría brindar pistas pertinentes en dicha tarea. 


\section{Referencias}

Aguirre, M. (2015). Los saberes tradicionales que poseen los campesinos de Guasca acerca de las semillas nativas. Tesis de Licenciatura en Biología no publicada, Universidad Pedagógica Nacional, Bogotá.

Barajas, Y., y Villamil, K. (2015). Reconocimiento de la apropiación de territorio de la población flotante de calle 72. Una mirada reflexiva hacia los comportamientos ambientales responsables como estrategia educativa. Tesis de Licenciatura en Biología no publicada, Universidad Pedagógica Nacional, Bogotá.

Barragán, B. (2011, Febrero-Mayo). Experiencia y narración: ensayo sobre el conocimiento escolar como campo de subjetivación. Revista Virtual Universidad Católica del Norte, No. 32.

Bejarano, D., y Mora, A. (2008). Una aproximación al estado de incorporación de la dimensión ambiental en el proyecto curricular de la Licenciatura en Biología de la Universidad Pedagógica Nacional. Tesis de Licenciatura en Biología no publicada, Universidad Pedagógica Nacional, Bogotá.

Bilbao, A., y Garzón, S. (2003). Creencias de los estudiantes de grado sexto del CED Luis López de Mesa acerca del concepto ambiente y sus implicaciones en la enseñanza de las ciencias. Tesis de Licenciatura en Biología no publicada, Universidad Pedagógica Nacional, Bogotá.
Cárdenas, J. (2015). Aproximación a la conformación discursiva de la biodiversidad en la escuela colombiana (2000-2010). Tesis de Licenciatura en Biología no publicada, Universidad Pedagógica Nacional, Bogotá.

Castro, J. (2007). La ficha analítica y la ficha temática: Soportes para el trabajo documental. Documento de trabajo. Bogotá.

Costanza, R., y Daly, H. (1992). Natural capital and sustainable development. Conservation Biology, 6(1). DOI: https://doi.org/10.1046/j.1523-1739.19 92.610037.x

Eschenhagen, M. (2009). Educación ambiental superior en América Latina, retos epistemológicos y curriculares. Bogotá: Biblioteca Universidad y Ambiente.

Eschenhagen, M. (2010-Mayo). Los límites de la retorica verde. Revista Gestión y Ambiente, Vol. 13, No. 1.

Gómez, C., y Acuña, W. (2009). La unidad didáctica como herramienta integradora de las relaciones existentes entre educación ambiental, desarrollo sustentable humano y ciencias naturales en el Instituto Departamental El Hato, sede Agua Dulce y Colegio Ignacio Pescador, Sede El Pulpito. Tesis de Licenciatura en Biología no publicada, Universidad Pedagógica Nacional, Bogotá. 
Gómez, M., y Bermúdez, E. (2015). Diagnóstico de la dimensión ambiental de la Universidad Pedagógica Nacional, sede calle 72. Aportes para la construcción del proyecto ambiental universitario. Licenciatura en Biología. Bogotá: Universidad Pedagógica Nacional.

González, C. (2017). La educación ambiental ante el problema ético del desarrollo. Educare, Vol. 21, No. 2. Obtenido desde https://dialnet.unirioja.es/ descarga/articulo/5919101.pdf DOI: https://doi. org/10.15359/ree.21-2.14

González, E. (2003). Educación para la ciudadanía ambiental. Interciencia, Vol. 28, No. 10. Obtenido desde https://www.researchgate. net/publication/322767862_Educacion_para_la_ ciudadania_ambiental

Gudynas, E. (2009). Ciudadanía ambiental y metaciudadanías ecológicas. Revisión y alternativas en América Latina. En Reyes, J., y Castro, E. Urgencia y utopía frente a la crisis de civilización. México: Universidad de Guadalajara. Obtenido desde http://www.ecologiasocial.com/biblioteca/ GudynasCiudadaniasMetaciudadaniasMx09x2.pdf

Larrosa, J. (2011). Experiencia y alteridad en educación. Rosario: Homo Sapiens Ediciones.

Lastra, D. (2013). El ambiente: asunto de sujetos y experiencias. Un acercamiento a la complejidad de lo ambiental en la escuela. Trabajo de Grado, Especialización en Pedagogía, no publicado, Universidad Pedagógica Nacional, Bogotá.
Lastra, D., y Ramírez, A. (2013). La enseñanza ambiental como propuesta de formación integral. Revista TED, No. 34. Bogotá: Universidad Pedagógica Nacional. Obtenido desde http:// www.scielo.org.co/pdf/ted/n34/n34a05.pdf DOI: https://doi.org/10.17227/01213814.34ted70.90

Leff, E. (2004). Racionalidad ambiental. La reapropiación social de la naturaleza. Buenos Aires: Siglo XXI.

Leff, E. (2007). La complejidad ambiental. Revista Virtual Gaia Scientia, Vol. 1, No. 1.

López, A., y Pérez, D. (2010). Contribución al fortalecimiento de valores ambientales en los estudiantes de grado décimo frente a la conservación de su ambiente natural, utilizando el Camino Real La Chucua como herramienta pedagógica. Tesis de Licenciatura en Biología no publicada, Universidad Pedagógica Nacional, Bogotá.

Michelini, D. (2007). Bien común y ética pública. Alcances y límites del concepto tradicional de bien común. Tópicos 1, No. 15. Santa Fe: Universidad Católica de Santa Fe.

Morin, E. (2007). Introducción al pensamiento complejo. Barcelona: Gedisa.

Paz, M. (2009). Educación ambiental y nuevos paradigmas: De la "otredad" ambiental a la complejidad en los procesos de aprendizaje. Trabajo presentado en el VI Congreso Iberoamericano de Educación Ambiental, 16 al 19 de Septiembre, San Clemente de Tuyú, Buenos Aires, Argentina. 
Obtenido desde https://www.researchgate.net/ publication/323486811_Educacion_ambiental_y_ nuevos_paradigmas_De_la_otredad_ambiental_a_ la_complejidad_en_los_procesos_de_aprendizaje /link/5a982750aca27214056d39b6/download

Piñeros. I. (2008). De paseo por la ciudad: una experiencia para el fortalecimiento de una ciudadanía ambiental. Tesis de Licenciatura en Biología no publicada, Universidad Pedagógica Nacional, Bogotá.

Poveda, D. (2009). Modelo de educación ambiental campesino: "educando en el saber y la autonomía campesina de Los Verjones". Tesis de Licenciatura en Biología no publicada, Universidad Pedagógica Nacional, Bogotá.

Rodríguez, A. (2011). Aproximación a la dinámica ambiental de los municipios de Leticia y Puerto Nariño (Amazonas), con miras a fundamentar el diseño e implementación de propuestas de educación ambiental para la región. Tesis de Licenciatura en Biología no publicada, Universidad Pedagógica Nacional, Bogotá

Sauvé, L. (1994). Exploración de la diversidad de conceptos y de prácticas en la educación relativa al ambiente. Memorias, Seminario Internacional: La dimensión ambiental y la escuela. Bogotá: Ministerio de Educación Nacional.

Torres, G. (2015). La pedagogía ambiental: hacia un nuevo paradigma educativo. Revista Entreciencias: diálogos en la Sociedad del Conocimiento, Vol. 3. No. 7. México: Universidad Nacional Autónoma de México. Obtenido desde https://www.redalyc. org/pdf/4576/457644945008.pdf DOI: https:// doi.org/10.21933/J.EDSC.2015.07.117

Valencia, O., y Tobar, J. (2003). Bio/ecocapitalismo y "reinvención de la emancipación social". Biopolítica y filosofías de vida. Cali: Editorial Universidad del Cauca.

Vargas, C. (2014). Posibilidades e imposibilidades de la incorporación de la educación ambiental en la escuela: propuesta de estrategia evaluativa. Revista Bio-Grafía, Vol. 7, No. 12. Bogotá: Universidad Pedagógica Nacional. Obtenido desde https:// revistas.pedagogica.edu.co/index.php/biografia/article/view/2855/2569 DOI: https://doi. org/10.17227/20271034.12biografia35.49

Zuluaga, O. (1999). Pedagogía e historia. La historicidad de la pedagogía, la enseñanza un objeto de saber. Antioquia: Anthropos. 